\title{
UNIFORM $\sigma$-ADDITIVITY IN SPACES OF BOCHNER OR PETTIS INTEGRABLE FUNCTIONS OVER A LOCALLY COMPACT GROUP
}

\author{
NICOLAE DINCULEANU
}

\begin{abstract}
If $G$ is an abelian locally compact group with Haar measure $\mu, E$ is a Banach space and $K \subset L_{F}^{\prime}(\mu)$, we give necessary and sufficient conditions for the set $\left\{\int_{1},|f| d \mu: f \in K\right\}$ to be uniformly $\sigma$-additive in terms of uniform convergence on $K$, for the topology $\sigma\left(L_{E}^{1}, L_{E^{\prime}}^{\propto}\right)$ of convolution and translation operators. In case $E=R$, this gives a new characterization of relatively weakly compact sets $K \subset l$.'
\end{abstract}

1. Introduction. In this paper we consider the space $L_{E}^{1}$ of Bochner integrable functions and the space $L_{E}^{P I}$ of Pettis integrable functions over an abelian locally compact group $G$ endowed with a Haar measure $\mu$, and we give a characterization of uniform $\sigma$-additivity in terms of uniform convergence-in the topology $\sigma^{\prime}=$ $\sigma\left(L_{E}^{1}, L_{E^{\prime}}^{\infty}\right)$, respectively in the weak topology of $\varrho_{E}^{1}-$ of convolution operators and translation operators. If $E=R$, this yields a new characterization of relative weak compactness in $L^{\prime}$.

The convolution of Bochner integrable functions has been studied in [2] and has been extended in [4] for Pettis integrable functions.

Similar results have been obtained in a previous paper [3], where we give a characterization of uniform $\sigma$-additivity in the spaces $L_{E}^{1}$ and $\mathcal{L}_{E}^{1}$ over a measure space $(X, \Sigma, \mu)$, in terms of uniform convergence, in the $\sigma^{\prime}$-topology or in the weak topology, of conditional expectations.

2. Uniform $\sigma$-additivity in the Lebesgue space $L_{E}^{1}$. Let $G$ be an abelian locally compact additive group endowed with a Haar measure $\mu$; let $E$ be a Banach space and $L_{E}^{\prime}$ be the space of Bochner $\mu$-integrable functions $f: G \rightarrow E$. For each relatively compact neighborhood $V$ of 0 in $G$, we choose a function $u_{V}$ on $G$ which is positive, bounded, symmetric (i.e. $u_{V}(-t)=u_{V}(t)$ ), vanishes outside $V$ and $\int u_{\nu} d \mu=1$. If $\mathscr{V}$ is a base of relatively compact neighborhoods of 0 in $G$, we call $\left(u_{V}\right)_{V \in \mathcal{V}}$ an approximate unit. We denote by $u_{V} * f$ the convolution: $u_{V} * f(t)=\int u_{V}(t-s) f(s) d s$, for $t \in G$. For $h \in G$ we denote by $T^{h}$ the translation operator, defined by $\left(T^{h} f\right)(t)=$ $f(t+h)$, for $t \in G$. If $f \in L_{E}^{1}$, we denote by $f \mu$ the measure defined for any Borel set $A \subset G$ by $(f \mu)(A)=\int_{A} f d \mu$. Finally, we denote by $\sigma^{\prime}$ the topology $\sigma\left(L_{E}^{1}, L_{E^{\prime}}^{\infty}\right)$ on

Received by the editors September 15, 1981.

1980 Mathematics Subject Classification. Primary 28B05; Secondary 43A20, 46E40, 46G10.

Key words and phrases. Locally compact group, Harr measure, Bochner integral, Pettis integral, convolution, translation, approximate unit, uniform $\sigma$-additivity, uniform convergence. 
$L_{E}^{1}$. If $E^{\prime}$ has the Radon-Nikodym property, then the $\sigma^{\prime}$-topology is the weak topology of $L_{E}^{\prime}$.

THEOREM 1. Let $K \subset L_{E}^{1}$ be a set.

I. The set $|K| \mu=\{|f| \mu ; f \in K\}$ is uniformly $\sigma$-additive, if and only if the following conditions are satisfied:

(a) $K$ is bounded in $L_{t}^{1}$;

(b) For every countable subset $K_{0} \subset K$, there exists a decreasing sequence $\left(V_{n}\right)$ of neighborhoods of 0 in $G$, such that either

$\left(\mathrm{b}_{1}\right) \lim _{n} u_{V_{n}} * f=f$ in $L_{E}^{1}$ for the $\sigma^{\prime}$-topolog $v$, uniformly for $f \in K_{0}$; or

$\left(b_{2}\right) \lim _{h \in V_{n}, n \rightarrow \infty} T^{h} f=f$, in $L_{E}^{1}$, for the $\sigma^{\prime}$-topology, uniformly for $f \in K_{0}$;

(c) $\lim _{C} \chi_{C} f=f$, strongly in $L_{E}^{1}$, uniformly for $f \in K$, where the limit is taken along the increasing net of all compact subsets of $G$.

(Condition (c) is superfluous if all functions of $K$ vanish outside a common compact set; in particular, if $G$ is compact.)

II. If $|K| \mu$ is uniformly $\sigma$-additive, then

$\left(\mathrm{b}_{1}^{\prime}\right) \lim _{V} u_{V} * f=f$ and

$\left(b_{2}^{\prime}\right) \lim _{h \rightarrow 0} T^{h} f=f$,

in $L_{E}^{1}$, for the $\sigma^{\prime}$-topology, uniformly for $f \in K$.

Proof. Assume first conditions (a), $\left(b_{1}\right)$ and (c) satisfied and prove that $|K| \mu$ is uniformly $\sigma$-additive. Let $C \subset G$ be a compact set and $\phi \in L^{1} \cap L^{\infty}$ a function with compact support $V$.

(A) The set $\phi *\left(\chi_{C} K\right)=\left\{\phi *\left(\chi_{C} f\right) ; f \in K\right\}$ is bounded in $L_{E}^{1}$. In fact. if $f \in K$. then

$$
\left\|\phi *\left(\chi_{C} f\right)\right\|_{1} \leqslant\|\phi\|_{1}\left\|\chi_{C} f\right\|_{1} \leqslant M\|\phi\|_{1},
$$

where $M=\sup \left\{\|f\|_{1} ; f \in K\right\}$.

(B) The set $\left|\phi *\left(\chi_{C} K\right)\right| \mu$ is uniformly $\sigma$-additive. In fact the set $\phi *\left(\chi_{C} K\right)$ is bounded in $L_{E}^{\infty}$ :

$$
\left\|\phi *\left(\chi_{C} f\right)\right\|_{\infty} \leqslant\|\phi\|_{\infty}\left\|\chi_{C} f\right\|_{1} \leqslant M\|\phi\|_{1}, \quad \text { for } f \in K .
$$

It follows that the set $\left|\phi *\left(\chi_{C} K\right)\right| \mu$ is uniformly absolutely $\mu$-continuous. Since all the functions of $\phi *\left(\chi_{C} K\right)$ vanish outside the compact set $C+V$, the set $\left|\phi *\left(\chi_{C} K\right)\right| \mu$ is uniformly $\sigma$-additive.

(C) For every $g \in L_{E^{\prime}}^{\infty}$ and $f \in K$ we have

$$
\begin{aligned}
\left|\int\left\langle\phi *\left(\chi_{C} f\right)-f, g\right\rangle d \mu\right| & \leqslant\left|\int\left\langle\phi *\left(\chi_{C} f-f\right), g\right\rangle d \mu\right|+\left|\int\langle\phi * f-f, g\rangle d \mu\right| \\
& \leqslant\|\phi\|_{1}\left\|\chi_{C} f-f\right\|_{1}\|g\|_{\infty}+\left|\int\langle\phi * f-f, g\rangle d \mu\right| .
\end{aligned}
$$

From condition (c) we deduce that there is an increasing sequence $\left(C_{n}\right)$ of compact sets such that $\lim _{n}\left\|\chi_{C_{n}} f-f\right\|_{1}=0$, uniformly for $f \in K$. Let $K_{0} \subset K$ be a countable set. Taking above $C=C_{n}$ and $\phi=u_{V_{n}}$, where $\left(V_{n}\right)$ is the sequence stated in condition (b), we deduce that

$$
\lim _{n} u_{V_{n}} *\left(\chi_{C_{n}} f\right)=f, \quad \text { in } L_{E}^{1}
$$


for the $\sigma^{\prime}$-topology, uniformly for $f \in K_{0}$. Since, for each $n$, the set $\left|u_{V_{n}}{ }^{*}\left(\chi_{C_{n}} K_{0}\right)\right| \mu$ is bounded and uniformly $\sigma$-additive, from Lemma lb in [3] we deduce that $\left|K_{0}\right| \mu$ is also uniformly $\sigma$-additive. Since $K_{0}$ was an arbitrary countable set in $K$, it follows that $|K| \mu$ is uniformly $\sigma$-additive. If conditions $(a),\left(b_{2}\right)$ and (c) are satisfied, then $|K| \mu$ is again uniformly $\sigma$-additive, since by Proposition 12 in [4]. condition $\left(b_{2}\right)$ implies $\left(b_{1}\right)$. We remark that in [4], the implication $b_{2} \Rightarrow b_{1}$ is stated for the weak topology. but the same proof is valid for the $\sigma^{\prime}$-topology.

Conversely, assume $|K| \boldsymbol{\mu}$ is uniformly $\sigma$-additive.

(D) $K$ is bounded in $L_{E}^{1}$. In fact, we can find a compact set $B \subset G$ such that $\int_{G-B}|f| d \mu \leqslant 1$ for all $f \in K$. Since $|K| \mu$ is uniformly absolutely $\mu$-continuous. there is $\eta>0$ such that if $\mu(A) \leqslant \eta$, then $\int_{A}|f| d \mu \leqslant 1$ for all $f \in K$. Since the Haar measure is diffuse, it has the Darboux property: there is a finite family of disjoint Borel sets $A_{1} \ldots \ldots A_{n}$ with union $B$, such that $\mu\left(A_{1}\right) \leqslant \eta$ for $i=1 \ldots \ldots n$. It follows that $\int_{B}|f| d \mu \leqslant n$ for all $f \in K$. hence $\int|f| d \mu \leqslant n+1$ for all $f \in K$ : consequently $K$ is bounded.

(E) Since $|K| \mu$ is uniformly $\sigma$-additive, for every $\varepsilon>0$ there is a compact set $C \subset G$ such that

$$
\int_{(i-c}|f| d \mu<\varepsilon, \quad \text { for all } f \in K
$$

that is

$$
\int\left|\chi_{c} f-f\right| d \mu<\varepsilon, \quad \text { for all } f \in K
$$

and condition (c) follows.

(F) Let $f \in L_{l}^{1}, g \in L_{l}^{x}, \lambda>0 . h \in G$, and $C$ be an integrable subset. Then

$$
\begin{aligned}
\left|\int\left\langle T^{h} f-f . g\right\rangle d \mu\right| \leqslant & 2\|g\|_{x} \int_{(i, c}|f| d \mu+2\|g\|_{x} \int_{\{\mid, \backslash \lambda\}}|f| d \mu \\
& +\lambda\left\|T^{-h}\left(\chi_{c} g\right)-\chi_{c} g\right\|_{1}+\lambda\|g\|_{\infty}\left\|\chi_{c}-\chi_{c}{ }_{h}\right\|_{1} .
\end{aligned}
$$

In fact

$$
\begin{aligned}
\left|\int\left\langle T^{h} f-f, g\right\rangle d \mu\right| \leqslant & \left|\int\left\langle T^{h}\left(f \chi_{(;-\ldots)}\right)-f \chi_{(;-\ldots, \cdot g}\right\rangle d \mu\right| \\
& +\left|\int\left\langle T^{h}\left(f \chi_{(\cdot)}\right)-f \chi_{(\because) g}\right\rangle d \mu\right| .
\end{aligned}
$$

The first term can be written

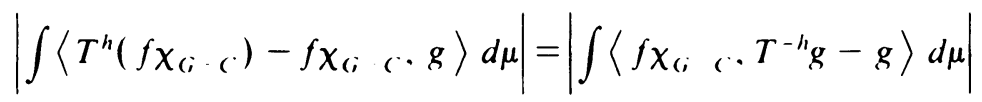

$$
\begin{aligned}
& \leqslant 2\|g\|_{x} \int_{(i-c i}|f| d \mu .
\end{aligned}
$$


For the second term we have $T^{h}\left(f \chi_{C}\right)=\chi_{C-h} T^{h}\left(f \chi_{C}\right)$, hence

$$
\begin{aligned}
\left|\int\left\langle T^{h}\left(f \chi_{C}\right)-f \chi_{C}, g\right\rangle d \mu\right|= & \left|\int\left\langle f \chi_{C}, T^{-h}\left(g \chi_{C-h}\right)-g \chi_{C}\right\rangle d \mu\right| \\
\leqslant & \left|\int_{C \cap\{(n)>\lambda\}}\left\langle f, T^{-h}\left(g \chi_{C-h}\right)-g \chi_{C}\right\rangle d \mu\right| \\
& +\left|\int_{C \cap\{|\eta| \lambda\}}\right|\left\langle f, T^{-h}\left(g \chi_{C-h}\right)-g \chi_{C}\right\rangle d \mu \\
\leqslant & 2\|g\|_{\infty} \int_{\{|\eta|>\lambda\}}|f| d \mu+\lambda\left\|T^{-h}\left(g \chi_{C-h}\right)-g \chi_{C}\right\|_{1} \\
\leqslant & 2\|g\|_{\infty} \int_{\{l n>\lambda\}}|f| d \mu+\lambda\left\|T^{-h}\left(g \chi_{C}\right)-g \chi_{C}\right\|_{1} \\
& +\lambda\left\|T^{-h} g\left(\chi_{C-h}-\chi_{C}\right)\right\|_{1}
\end{aligned}
$$

and this last term is smaller than $\lambda\|g\|_{\infty}\left\|\chi_{C-h}-\chi_{C}\right\|_{1}$.

(G) We can now prove conditions $\left(b_{2}^{\prime}\right)$ and $\left(b_{1}^{\prime}\right)$. Let $g \in L_{E^{\prime}}^{x}, g \neq 0$ and $\varepsilon>0$. Take $C \subset G$ such that

$$
\int_{G-C}|f| d \mu<\varepsilon /\left(8\|g\|_{\infty}\right), \quad \text { for all } f \in K .
$$

Take also $\lambda>0$ such that

$$
\int_{\{(n>\lambda\}}|f| d \mu<\varepsilon /\left(8\|g\|_{\infty}\right), \quad \text { for all } f \in K .
$$

We can find a symmetric neighborhood $V$ of 0 such that for all $h \in V$ we have

$$
\left\|T^{-h}\left(\chi_{C} g\right)-\chi_{C} g\right\|_{1}<\varepsilon / 4,
$$

and

$$
\left\|\chi_{C}-\chi_{C-h}\right\|_{1}=\left\|\chi_{C}-T^{h} \chi_{C}\right\|_{1}<\varepsilon /\left(4 \lambda\|g\|_{\infty}\right) .
$$

Then, for $h \in V$ and all $f \in K$ we have, from step (F),

$$
\left|\int\left\langle T^{h} f, g\right\rangle d \mu\right|<\varepsilon
$$

that is $\lim _{h \rightarrow 0} T^{h} f=f$, in $L_{E}^{\prime}$ for the $\sigma^{\prime}$-topology, uniformly for $f \in K$. This proves condition $\left(b_{2}^{\prime}\right)$; and condition $\left(b_{1}^{\prime}\right)$ follows from Proposition 12 in [3].

(H) To prove condition $\left(\mathrm{b}_{2}\right)$, let $K_{0} \subset K$ be a countable subset. The proof of condition $\left(b_{1}\right)$ is the same as in step $(G)$.

Let $R_{0}$ be a countable ring of relatively compact Borel subsets of $G$, such that any function of $K_{0}$ is the limit $\mu$-a.e. and in $L_{E}^{1}$ of step functions over $R_{0}$.

Since for each $f \in L_{E}^{1}$ we have $\lim _{h \rightarrow 0} T^{h} f=f$, strongly in $L_{E}^{1}$, we can find a decreasing sequence $\left(V_{n}\right)$ of symmetric neighborhoods of 0 , such that $\lim _{h \in V_{n}, n \rightarrow \infty} T^{h} \chi_{A}=\chi_{A}$, strongly in $L^{\prime}$ for every $A \in R_{0}$. Next, we choose arbitrarily a sequence $\left(h_{n}\right)$ such that $h_{2 n-1}=-h_{2 n} \in V_{n}$ for every $n$. Then $\lim _{n} T^{h_{n}} \chi_{A}$ $=\chi_{A}$ strongly in $L^{\prime}$ for every $A \in R_{0}$. Consider the group $\Gamma \subset G$ generated by the 
sequence $\left(h_{n}\right)$. Then the set $L$ of linear combinations of functions of the form $\left(T^{\alpha_{1}} \chi_{A_{1}}\right)\left(T^{\alpha_{2}} \chi_{A_{2}}\right) \cdots\left(T^{\alpha_{k}} \chi_{A_{k}}\right)$ with $\alpha_{1}, \ldots, \alpha_{k} \in \Gamma$ and $A_{1}, \ldots, A_{k} \in R_{0}$, is an algebra of $\mu$-integrable functions, invariant with respect to $T^{\alpha}$ for any $\alpha \in \Gamma$. Moreover, $\lim _{n} T^{h_{n}} \chi=\chi$, in $L^{1}$, for all $\chi \in L$.

It is enough to check this for the functions of the form $\chi=\left(T^{\alpha_{1}} \chi_{A_{1}}\right)\left(T^{\alpha_{2}} \chi_{A_{2}}\right)$. We have $\lim _{n} T^{h_{n}} \chi=\chi, \mu$-a.e. and since $\left|T^{h_{n}} \chi\right| \leqslant \chi_{v_{1}}+\left(A_{1} \cup A_{2}\right)$, we can apply Lebesgue's dominated convergence theorem and deduce that $\lim _{n} T^{h_{n}} \chi=\chi$ in $L^{1}$.

Moreover, this last equality remains valid for $\chi$ in the closure of $L$ in $L^{\prime}$, since $\sup _{n}\left\|T^{h_{n}}\right\|=1$.

The class $\Lambda=\left\{A ; \chi_{A} \in L\right\}$ is a ring containing $R_{0}$, and the class $\left\{\chi_{A} ; A \in \Lambda\right\}$ is invariant with respect to $T^{\alpha}$ for all $\alpha \in \Gamma$. All functions of $L$ vanish $\mu$-a.e. outside a o-finite set $X_{0}$.

The $\delta$-ring $\Sigma_{0}$ generated by $\Lambda$ is the completion of $\Lambda$ for the semidistance $\rho(A, B)=\mu(A \Delta B)=\left\|\chi_{A}-\chi_{B}\right\|_{1}$, and can be obtained-modulo negligible sets - as closure in $L^{1}$ of the set of functions $\chi_{A}$ with $A \in \Lambda$. It follows that the class $\left\{\chi_{A} ; A \in \Sigma_{0}\right\}$ is invariant with respect to $T^{\alpha}$ for all $\alpha \in \Gamma$, since the class $\left\{\chi_{A} ; A \in\right.$ $\Lambda$ \} has this property, and since this property is preserved by passing to limits in $L^{\prime}$.

We deduce then that for any Banach space $F$, the space $L_{F}^{1}\left(X_{0}, \Sigma_{0}, \mu\right)$ is invariant with respect to $T^{\alpha}$ for all $\alpha \in \Gamma$, and that for every $f \in L_{F}^{1}\left(X_{0}, \mu\right)$ we have $\lim _{n} T^{h_{n}} f=f$, strongly in $L_{F}^{\prime}\left(X_{0}, \Sigma_{0}, \mu\right)$.

In fact this property is valid for all step functions, and $\sup _{n}\left\|T^{h_{n}}\right\|=1$.

We are now ready to prove condition $\left(b_{2}\right)$.

Let $g \in L_{E^{\prime}}^{\infty}, g \neq 0$, and $\varepsilon>0$. The conditional expectation $g^{\prime}=E\left(g \mid \Sigma_{0}\right)$ is defined since the space $(G, \mu)$ is localizable (see [1]).

We can consider $g^{\prime} \in L_{E^{\prime}}^{\infty}\left(X_{0}, \Sigma_{0}, \mu\right)$. Since $K_{0} \subset L_{E}^{1}\left(X_{0}, \Sigma_{0}, \mu\right)$ and $\left|K_{0}\right| \mu$ is uniformly $\sigma$-additive, there is a set $C \in \Sigma_{0}$ such that

$$
\int_{G-C}|f| d \mu<\varepsilon /\left(8\|g\|_{\infty}\right), \quad \text { for all } f \in K_{0} .
$$

Also let $\lambda$ be such that

$$
\int_{\{(n>\lambda)}|f| d \mu<\varepsilon /\left(8\|g\|_{\infty}\right), \quad \text { for all } f \in K_{0} .
$$

Since $\chi_{C} g^{\prime} \in L_{E^{\prime}}^{\prime}\left(X_{0}, \Sigma_{0}, \mu\right)$, we have $\lim _{n} T^{h_{n}}\left(\chi_{C} g^{\prime}\right)=\chi_{C} g^{\prime}$, strongly in $L_{E^{\prime}}^{\prime}$; and we have also

$$
\lim _{n}\left\|\chi_{C}-\chi_{C-h_{n}}\right\|_{1}=\lim _{n}\left\|\chi_{C}-T^{h_{n}} \chi_{C}\right\|_{1}=0
$$

Let $n_{\varepsilon}$ be such that for $n \geqslant n_{\varepsilon}$ we have

$$
\left\|T^{h_{n}}\left(\chi_{C} g^{\prime}\right)-\chi_{C} g^{\prime}\right\|_{1}<\varepsilon /(4 \lambda)
$$

and

$$
\left\|\chi_{c}-\chi_{C-h}\right\|_{1}<\varepsilon /\left(4 \lambda\|g\|_{\infty}\right)
$$


Then, for any $f \in K_{0}$ and any $n \geqslant n_{\text {s }}$ we deduce from step (F),

$$
\left|\int\left\langle T^{h_{n}} f, g\right\rangle d \mu\right|=\int\left\langle T^{h} f, g^{\prime}\right\rangle d \mu \mid<\varepsilon,
$$

that is $\lim _{n} T^{h_{n}} f=f$, in $L_{E}^{1}$ for the $\sigma^{\prime}$-topology, uniformly for $f \in K_{0}$. Since the sequence $\left(h_{n}\right)$ was arbitrary, it follows that

$$
\lim _{h \in V_{n}^{\prime}, n \rightarrow \infty} T^{h} f=f, \quad \text { in } L_{K}^{1}
$$

for the $\sigma^{\prime}$-topology, uniformly for $f \in K$ and so, condition $\left(\mathrm{b}_{2}\right)$ is proved. Condition $\left(b_{1}\right)$ then follows from Proposition 12 in [4]; and this completes the proof of the theorem.

Remark. The $\sigma^{\prime}$-topology cannot be replaced by the weak topology. There are examples (to be published in a joint paper with Jürgen Batt) of relatively weakly compact sets $K \subset L_{E}^{1}$ over the circle group, such that the limits in $\left(\mathrm{b}_{1}\right)$ and $\left(\mathrm{b}_{2}\right)$ for the weak topology are false.

3. Uniform $\sigma$-additivity in the Pettis space $E_{E}^{\prime}$. We denote by $\mathscr{L}_{E}^{\prime}$ the Pettis space of functions $f: G \rightarrow E$ which are strongly $\mu$-measurable and Pettis integrable, endowed with the Pettis norm

$$
(f)_{1}=\sup \left\{\int\left|\left\langle f, x^{\prime}\right\rangle\right| d \mu: x^{\prime} \in E_{1}^{\prime}\right\}
$$

where $E_{\mathrm{1}}^{\prime}$ is the unit ball of $E^{\prime}$. A set $F \subset E_{\mathrm{1}}^{\prime}$ is norming for a set $K \subset E_{E}^{\prime}$. if $|f(t)|=\sup \left\{\left|\left\langle f(t), x^{\prime}\right\rangle\right| ; x^{\prime} \subset F\right\}, \mu$-a.e. for every $f \in K$.

THEOREM 2. Let $K \subset L_{E}^{\prime}$ be a set.

1. The set $K \mu$ is uniformly $\sigma$-additive, if and only if the following conditions are satisfied:

(a) $K$ is bounded in $\mathrm{L}_{E}^{\prime}$;

(b) For every countable subset $K_{0} \subset K$ there is a decreasing sequence $\left(V_{n}\right)$ of neighborhoods of 0 and a countable subset $E_{0}^{\prime} \subset E_{1}^{\prime}$, norming for $K_{0}$, such that either

$\left(b_{1}\right) \lim _{n}\left\langle u_{v_{n}} * f, x^{\prime}\right\rangle=\left\langle f, x^{\prime}\right\rangle$, weakly in $L^{\prime}$, uniformly for $f \in K_{0}$ and $x^{\prime} \in E_{0}^{\prime}$; or

$\left(\mathrm{b}_{2}\right) \lim _{h \in V_{n}, n \rightarrow \infty}\left\langle T^{h} f, x^{\prime}\right\rangle=\left\langle f, x^{\prime}\right\rangle$, weakly in $L^{\prime}$, uniformly for $f \in K_{0}$ and $x^{\prime} \in E_{0}^{\prime}$;

(c) $\lim _{C} f \chi_{C}=f$, strongly in $\mathcal{L}_{E}^{1}$, uniformly for $f \in K$, the limit being taken along the increasing net of all compact subsets of $G$.

II. If $K \mu$ is uniformly $\sigma$-additive, then:

$\left(b_{1}^{\prime}\right) \lim _{v}\left\langle u_{v} * f, x^{\prime}\right\rangle=\left\langle f, x^{\prime}\right\rangle$ and

$\left(\mathrm{b}_{2}^{\prime}\right) \lim _{h \rightarrow 0}\left\langle T^{h} f, x^{\prime}\right\rangle=\left\langle f, x^{\prime}\right\rangle$

weakly in $L^{\prime}$, uniformly for $f \in K$ and $x^{\prime} \in E_{1}^{\prime}$.

Proof. Assume first conditions (a), (b) and (c) satisfied. Let $K_{0} \subset K$ be a countable set, and $E_{0}^{\prime}$ the set corresponding to $K_{0}$ by condition (b) above.

Then the set $\left\langle K_{0}, E_{0}^{\prime}\right\rangle=\left\{\left\langle f, x^{\prime}\right\rangle ; f \in K_{0}, x^{\prime} \in E_{0}^{\prime}\right\}$ is countable and satisfies conditions (a), (b) and (c) of Theorem 1 , in the space $L^{1}$. It follows that $\left\langle K_{0}, E_{0}^{\prime}\right\rangle \mu$ is 
uniformly $\sigma$-additive; and then $K_{0} \mu$ is also uniformly $\sigma$-additive; therefore $K \mu$ is uniformly $\boldsymbol{\sigma}$-additive.

Conversely, assume $K \mu$ is uniformly $\sigma$-additive; let $K_{0} \subset K$ be countable, and let $E_{0}^{\prime} \subset E_{1}^{\prime}$ be a countable set, norming for $K_{0}$. The set $\left\langle K_{0}, E_{0}^{\prime}\right\rangle$ is countable and uniformly $\sigma$-additive. From Theorem 1 we deduce:

(a) The set $\left\langle K_{0}, E_{0}^{\prime}\right\rangle$ is bounded in $L^{1}$; hence $K_{0}$ is bounded in $L_{L}^{\prime \prime}$; therefore $K$ is bounded in ${ }^{\prime} !$;

(b) There exists a decreasing sequence $\left(V_{n}\right)$ of neighborhoods of 0 , satisfying conditions $\left(b_{1}\right)$ and $\left(b_{2}\right)$ of this theorem;

(c) $\lim _{C}\left\langle x_{C} f, x^{\prime}\right\rangle=\left\langle f, x^{\prime}\right\rangle$, strongly in $L^{\prime}$ uniformly for $f \in K$ and $x^{\prime} \in E_{0}^{\prime}$. which is equivalent to condition (c) of this theorem.

Finally, to obtain $\left(b_{1}^{\prime}\right)$ and $\left(b_{2}^{\prime}\right)$ we apply the second part of Theorem 1 to the set $\left\langle K, E_{\mathrm{l}}^{\prime}\right\rangle$.

Note. We take this opportunity to mention that Theorems 2(iii) and 4(iii) in [3] are valid without the condition $\sup \{|f(t)| ; f \in K\}<\infty$, $\mu$-a.e. The proof will be given in a forthcoming paper, for a more general situation.

\section{REFERENCES}

1. N. Dinculcanu. (onditional expectattons for general measure space. J. Multivariate Anal 1 (1971). $347-364$

2. Integration on lecalh compact spaces. Noordhoff. Levden. 1974

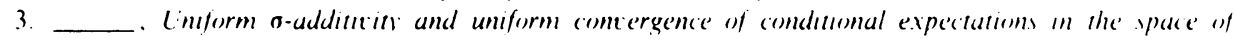
Bochner or Pettis integrable functions. (ieneral Topology and Modern Analysis. Acadenic Press. New York. 1980). pp. 391-397.

4. . On Kolmogoron-Tamarkin and M. Riesz strong compactness criteria in functum spaces ore' a locally compact group. J. Math. Anal. Appl. (to appear).

Dipartmint of Mathimatics, University of Fi.orid)a. (iainisvili.i. Fiorid) 32611 